OPEN ACCESS

Edited by:

Peter Reiss,

Commissariat à l'Energie Atomique et aux Energies Alternatives (CEA),

France

Reviewed by:

Pengfei Qiu,

Shanghai Institute of Ceramics (CAS),

China

Andreu Cabot

Institut de Recerca de l'Energia de

Catalunya, Spain

*Correspondence:

Jianping Yang

jianpingyang@dhu.edu.cn

Wei Dai

daiwei@hue.edu.cn

Specialty section:

This article was submitted to

Energy Materials,

a section of the journal

Frontiers in Materials

Received: 04 August 2020

Accepted: 06 October 2020

Published: 28 October 2020

Citation:

Chen X, Yang J, Katkus T, Wu T, Tao J, Li J, Wang C, Wang $X$ and Dai W (2020) Exploring Thermoelectric

Property Improvement for Binary

Copper Chalcogenides.

Front. Mater. 7:589568.

doi: 10.3389/fmats.2020.589568

\section{Exploring Thermoelectric Property Improvement for Binary Copper Chalcogenides}

\author{
Xinqi Chen ${ }^{1}$, Jianping Yang ${ }^{2 *}$, Tomas Katkus ${ }^{3}$, Tian $W^{1}{ }^{1}$, Junhui Tao ${ }^{1}$, Jie $\mathrm{Li}^{1}$, \\ Chuanhui Wang ${ }^{1}$, Xin Wang ${ }^{4}$ and Wei Dai ${ }^{1 *}$
}

${ }^{1}$ School of Physics and Mechanical \& Electrical Engineering, Hubei Engineering Technology Research Center of Environmental Purification Materials, Hubei University of Education, Wuhan, China, ${ }^{2}$ State Key Laboratory for Modification of Chemical Fibers and Polymer Materials, College of Materials Science and Engineering, Donghua University, Shanghai, China, ${ }^{3}$ Faculty of Science, Engineering and Technology, Swinburne University of Technology, Hawthorn, Victoria, Australia, ${ }^{4}$ School of Chemical and Biomedical Engineering, Nanyang Technological University, Singapore, Singapore

Copper chalcogenides have been the subject of extensive research as promising candidates in thermoelectric conversion applications due to their advantageous properties, environmental compatibility, and abundance. The low intrinsic thermal conductivity and high electric conductivity of these materials are born out of the "phonon-liquid electron-crystal" structure between the copper and chalcogens. In this review, strategies for thermoelectric property improvement of copper chalcogenides are discussed. The challenges and the future development of binary copper chalcogenides for further thermoelectric conversion applications are outlined.

Keywords: thermoelectric property, nanostructure, thermal conductivity, carrier concentration, flexible materials

\section{INTRODUCTION}

Solid-state thermoelectric (TE) technology, using the Seebeck effect and Peltier effect, can convert directly and simply between heat and electricity (Figure 1A) (Wei et al., 2018). The dimensionless TE figure of merit $(z T)$ is the key metric used to evaluate the performance of TE materials: $z T=S^{2}{ }_{\sigma} T / \kappa$. Where $S, \sigma, \kappa$, and $T$ are Seebeck coefficient, electrical conductivity, thermal conductivity, and absolute temperature, respectively (Tan et al., 2016). The earliest studies in TE technology are derived from the 19th century (Shi et al., 2016). Copper sulfides was reported as a promising TE material in 1827. However, there were two main problems unsolved in practical application, which are the stable composition of $\mathrm{Cu}_{2} \mathrm{~S}$ in reheating and contact between the $\mathrm{Cu}_{2} \mathrm{~S}$ and the electrode in the TE generator. When the "phonon-liquid electron-crystal" (PLEC) concept was proposed in 2012, the low thermal conductivity and high TE performance of copper sulfides and copper selenides have been explained. In this review, we discuss the strategies for TE property improvement of copper chalcogenides based on reducing lattice thermal conductivity of single-component material and tuning compositions for optimizing TE parameters. Challenges for binary copper chalcogenides and perspectives on the flexible copper chalcogenides are outlined.

\section{STRATEGIES FOR THERMOELECTRIC PROPERTY IMPROVEMENT}

The fundamental challenge of designing high-performance TE materials stems from the strong correlation of $S$, $\sigma$, and $\kappa$, which requires large $S$ and $\sigma$ values, as well as a low $\kappa$ value simultaneously 
A

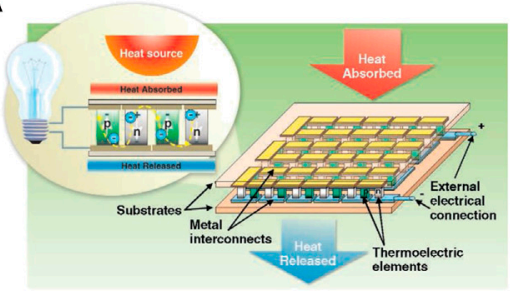

D

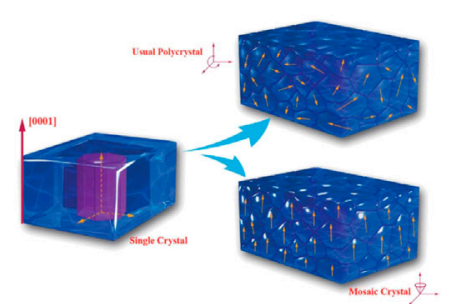

$\mathbf{F}$

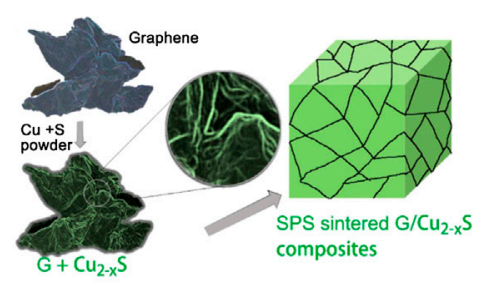

B

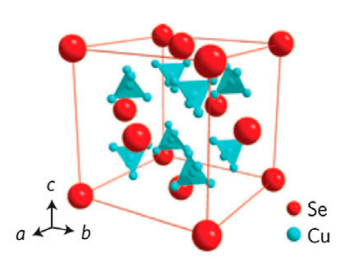

C

[111]

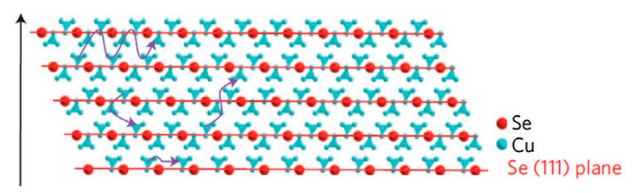

E

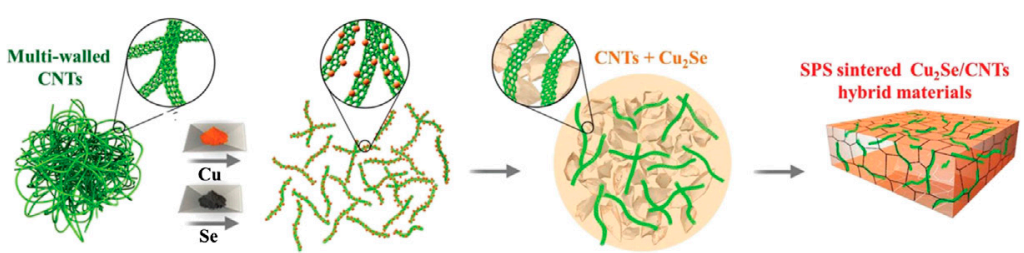

G

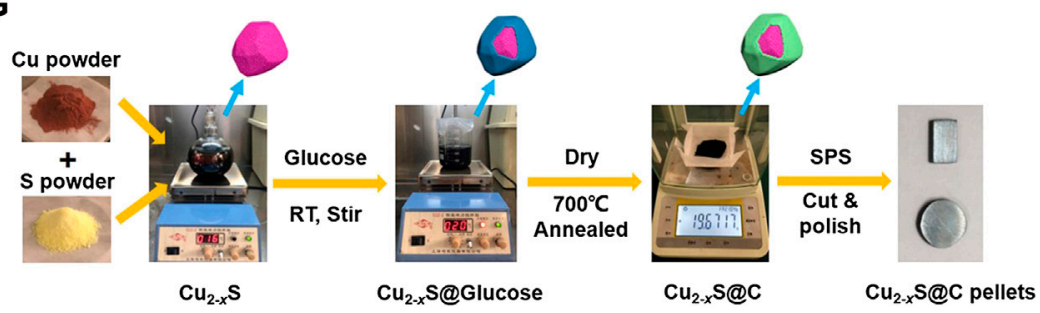

Figure 1 | (A) A thermoelectric generator module and heat drives (inset). Reproduced with permission (Service, 2004). Copyright 2004, American Association for the Advancement of Science. (B) Unit cell of cubic $\mathrm{Cu}_{2} \mathrm{Se}$. (C) Projected plane representation of the crystal structure along the cubic [1 -1 0] direction. The arrows indicate that the $\mathrm{Cu}$ ions can freely travel among the interstitial sites. Reproduced with permission (Liu et al., 2012). Copyright 2012, Nature Publication Group. (D) Structural characteristics of a single crystal, a usual polycrystal, and a mosaic crystal. Arrows refer to alignment of crystal grains. Reproduced with permission (He et al., 2015a). Copyright 2015, Wiley-VCH. (E) Fabrication process and formation mechanism of the Cu $2 \mathrm{Se} / \mathrm{CNT}$ hybrid materials. Reproduced with permission (Nunna et al., 2017). Copyright 2017, The Royal Society of Chemistry. (F) Process for fabricating G/Cu 2-x S composite. Reproduced with permission (Tang et al., 2018). Copyright 2018, Elsevier. (G) Process for fabricating carbon-encapsulated $\mathrm{Cu}_{2-x} \mathrm{~S}$ composite. Reproduced with permission (Chen et al., 2018). Copyright 2018, The Royal Society of Chemistry.

(Zhou et al., 2018). However, these three parameters are strongly interdependent through carrier concentration $(n)$. Among three parameters, $\kappa$ comprises the electronic thermal conductivity $\left(\kappa_{e}\right)$ and the lattice thermal conductivity $\left(\kappa_{L}\right)(\kappa=$ $\left.\kappa_{e}+\kappa_{L}\right) . \kappa_{L}$, calculated by $\kappa_{L}=1 / 3 C_{V} v l$, in which $C_{V}$ is heat capacity, $v$ is speed of sound, and $l$ is mean free path of phonons, is the only one with little dependence on $n$. Therefore, $\kappa_{L}$ can be minimized through the independent crystal structure and/or microstructure design. This is one strategy for TE property improvement. The other strategy, tuning the composition, such as foreign elements introducing, metal chalcogenides mixing, hybrid compounds building, strongly relates to the $n$ and carrier mobility $(\mu)$. Below we will briefly elaborate on these two strategies.

\section{Reducing Lattice Thermal Conductivity of Single Component Material}

Reducing $\kappa_{L}$ can rely on decreasing the mean free path of lattice phonons and eliminating some of the vibrational modes. For example, face-centered cubic (fcc) copper selenide, as a superionic conductor, has two different sub-lattices inside its crystal structure. The sub-lattice frame of Se atoms provides a pathway for the movement of semiconducting electrons, and the highly disordered $\mathrm{Cu}$ ions move through the Se lattice frame with "liquid-like mobility" and high diffusivities, which explained as the "PLEC" concept (Figures 1B and 1C) (Qiu et al., 2016; Chen et al., 2018). In addition, the liquid-like mobile $\mathrm{Cu}$ ions also introduce extra scattering to lattice phonons to further disrupt heat propagation or alter phonon transfer, and thereby shortening phonon mean free path and suppressing $\kappa_{L}$.

Now, research on the PLEC Cu-based superionic conductors has become a topic that is very popular in the TE community. A low $\kappa_{L}$ around $0.4-0.6 \mathrm{~W} \mathrm{~m}^{-1} \mathrm{~K}^{-1}$ was reported at a high-temperature of $1000 \mathrm{~K}$ for $\mathrm{Cu}_{2-x} \mathrm{Se}$, which leads to an enhanced TE performance $(z T=$ 1.5) (Liu et al., 2012). This high $z T$ value results from the coexistence of $\mathrm{Se}$ and $\mathrm{Cu}$ sub-lattices inside the crystal structure of the $\mathrm{Cu}_{2-x} \mathrm{Se}$ phase. Introducing nanostructure for defects creation, an effective method to enhance the low-to-high wavelength phonon scattering, results in 
a very small value of $\kappa . \mathrm{Cu}_{2} \mathrm{Se}$ with this low $\kappa\left(0.34 \mathrm{Wm}^{-1} \mathrm{~K}^{-1}\right)$ reached a high $z T$ of 2.1 at $973 \mathrm{~K}$ (Gahtori et al., 2015).

Copper sulfide and copper telluride are superionic conductors at high-temperatures as well. Thus, they correlate well with the PLEC character that has been observed in $\mathrm{Cu}_{2} \mathrm{Se}$. It is reported that extremely low values of $\kappa_{L}$ below $0.35 \mathrm{~W} \mathrm{~m}^{-1} \mathrm{~K}^{-1}$ and high values of $z T$ of 1.7 at $1000 \mathrm{~K}$ for $\mathrm{Cu}_{2-x} \mathrm{~S}$ with a slight copper deficiency (He et al., 2014). Copper telluride has an extremely high $\kappa_{e}$, therefore the $\kappa$ is also quite high compared with that in copper selenide and copper sulfide. For example, the value of $\kappa=2 \mathrm{~W} \mathrm{~m}^{-1} \mathrm{~K}^{-1}$ at room temperature and reduced to $1 \mathrm{~W} \mathrm{~m}^{-1} \mathrm{~K}^{-1}$ by increasing the annealing temperatures of samples, and the best $z T$ value is 1.1 at $1000 \mathrm{~K}$ (He et al., 2015b).

\section{Tuning Compositions for Optimizing Thermoelectric Parameters}

Tuning the composition, without the intrinsic restriction from monomers, more depends on the $n$ and $\mu$ to optimizing three TE parameters $S, \sigma$, and $\kappa$. Firstly, foreign elements introducing by the fabrication process can generate vacancies in material structures which increase the hole concentration at cation positions or electron concentration at cation positions. Furthermore, foreign elements serve as point-defect scattering sources for phonons. These impurity atoms create both force differences (strain field fluctuations) and mass contrast (mass fluctuations) between the host atoms and the impurity atoms and are very effective for scattering high-frequency (short wavelength) phonons. Thus foreign elements, like $\mathrm{Na}, \mathrm{Ag}, \mathrm{I}$, and $\mathrm{Al}$, can affect TE properties by changing the degree of $\mathrm{Cu}$ deficiency. For example, $\mathrm{Cu}_{2} \mathrm{Se}_{1-x} \mathrm{I}_{x}$ presents a $z T$ value of 2.3 near room temperature as a result of critical scattering, which simultaneously leads to a high $S$ and high $\kappa_{e}$ (Liu et al., 2013).

Another attractive way is the mixture of homogeneous metal chalcogenides, which not only maintains the PLEC cubic structure of copper chalcogenides but also creates a unique composite structure. He et al. reported a mosaic crystal of mixed $\mathrm{Cu}_{2} \mathrm{~S}-\mathrm{Cu}_{2} \mathrm{Te}$ bulk nanomaterials, which achieves a $z T$ value of 2.1 at $1000 \mathrm{~K}$ ( $\mathrm{He}$ et al., 2015a). In this unique microstructure, electrons are freely transferred along with the frames of quasi-single crystals while phonons are strongly scattered by lattice strains or interfaces of mosaic nanograins (Figure 1D). In the $\mathrm{Ag}_{2} \mathrm{Te}-\mathrm{Cu}_{2} \mathrm{Te}$ mixed structure, the high $n$ in pristine $\mathrm{Cu}_{2} \mathrm{Te}$ is substantially reduced to an optimal range, resulting in much-reduced $\kappa$ and enhanced power factors over the entire temperature range. Furthermore, the temperature of the last phase transition is significantly lowered when increasing $\mathrm{Ag}$ content, which leads to a $z T$ of 1.8 is achieved at $1000 \mathrm{~K}$ in $\mathrm{Cu}_{2} \mathrm{Te}+50 \% \mathrm{Ag}_{2} \mathrm{Te}$ (Zhao et al., 2019).

Recently, the introduction of different forms of carbon (such as carbon nanotubes, graphene, and carbon encapsulation) into the copper chalcogenide bulks has achieved significant enhancement of TE properties. Carbon brings to high charge $\mu$ into a composite structure (Zhu et al., 2019), which benefits the $\kappa_{e}$ but increases the $\kappa_{L}$ as well. The introduction of a certain concentration of carbon in composite materials is the key to improve the TE properties. $\mathrm{Cu}_{2} \mathrm{Se}$ and multi-walled carbon nanotube (CNTs) hybrid materials, with a high degree of homogeneously dispersed molecular CNTs inside the $\mathrm{Cu}_{2} \mathrm{Se}$ matrix, possess greatly improved $z T$ of 2.4 at $1000 \mathrm{~K}$ for $\mathrm{Cu}_{2} \mathrm{Se} / 0.75 \mathrm{wt} \% \mathrm{CNTs}$, which is about $30 \%$ higher than that of the pristine $\mathrm{Cu}_{2} \mathrm{Se}$ (Figure 1E) (Nunna et al., 2017). $\mathrm{Cu}_{2-x} \mathrm{~S}$ matrix and three-dimensional graphene heterointerface composites have created more interfaces between $\mathrm{Cu}_{2-x} \mathrm{~S}$ and graphene leading to low energy carrier scattering, which can increase the $S$ and cut down the $\kappa$. The $z T$ value of 1.56 at $873 \mathrm{~K}$ is in the $\mathrm{Cu}_{2-x} \mathrm{~S}$ with $0.75 \mathrm{wt} \%$ graphene (Figure 1F) (Tang et al., 2018). Carbonencapsulated $\mathrm{Cu}_{2-x} \mathrm{~S}$ composites have a $50 \%$ improvement of the $\kappa_{e}$ and power factor than that of the pristine $\mathrm{Cu}_{2-x} \mathrm{~S}$. The phase transition from orthorhombic to tetragonal of these composites demonstrated the lower $\kappa_{L}$. A $z T$ value of 1.04 has been achieved at $773 \mathrm{~K}$ in the $0.25 \mathrm{wt} \%$ carbon-encapsulated $\mathrm{Cu}_{2-x} \mathrm{~S}$ composites (Figure 1G) (Chen et al., 2019).

\section{CHALLENGES FOR BINARY COPPER CHALCOGENIDES}

$\mathrm{Cu}$ ions may migrate from their initial locations to places where electrical potential or chemical potential is low, thus the stabilities of the copper chalcogenides system still worth discussion ( $\mathrm{Ge}$ et al., 2016). In Dennler's experiments, it showed that $\mathrm{Cu}_{2} \mathrm{~S}$ is not electrically stable because both cracks and copper whiskers were observed in the sample after extended current stress testing (Figure 2A) (Dennler et al., 2014). Qiu et al. revealed the mechanism for atom migration and deposition in $\mathrm{Cu}-\mathrm{S} / \mathrm{Se}$ materials based on a critical chemical potential difference (Qiu et al., 2018). Constructing a series of electronically conducting but ion-blocking barriers to reset the chemical potential of such conductors to keep it below the threshold for decomposition can remain good stability under high electric currents and/or large temperature differences (Mao et al., 2019a). For example, high $z T$ and large critical voltage can be decoupled through doping immobile ions into the liquid-like sublattice (Mao et al., 2019b). In recent reports, the $\mathrm{Cu}_{2} \mathrm{Se} / \mathrm{Yb}_{0.3} \mathrm{Co}_{4} \mathrm{Sb}_{12} \mathrm{TE}$ module shows good stability and high energy conversion efficiency of 9.1\% (Figure 2B) (Qiu et al., 2019).

Flexible or thin-film TE materials can make up for application limitations from bulk materials, especially for light and portable devices. Several attempts have been used in the fabrication of flexible or thin-film copper chalcogenide structures. Typically, $\mathrm{Cu}_{2} \mathrm{Se}$ powder and the organic solution mixed making an ink solution, which was covered on flexible substrates evenly (Figures 2C and $\mathrm{D}$ ). The $\mathrm{Cu}_{2} \mathrm{Se}$ thin film fabricated by this route exhibits a power factor of $0.62 \mathrm{~mW} \mathrm{~cm}^{-1} \mathrm{~K}^{-2}$ at $684 \mathrm{~K}$ (Lin et al., 2017). Besides, $\mathrm{CdS} / \mathrm{Cu}_{2} \mathrm{~S}$ superlattice nanowires shown the enhanced TE performance. For measurement, $\mathrm{CdS} / \mathrm{Cu}_{2} \mathrm{~S}$ superlattice nanowires electrically connected to two electrodes that also serve as the temperature sensors. With a temperature gradient $(\Delta T)$ created by a DC current over a nearby heating electrode, a thermal voltage can be generated along the nanowire and the thermopower can be calculated (Figures 2E and F) (Xiong et al., 2017). 
A

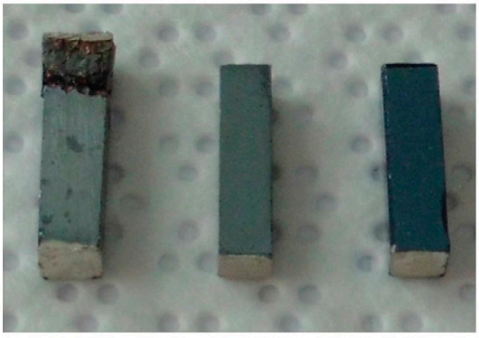

C

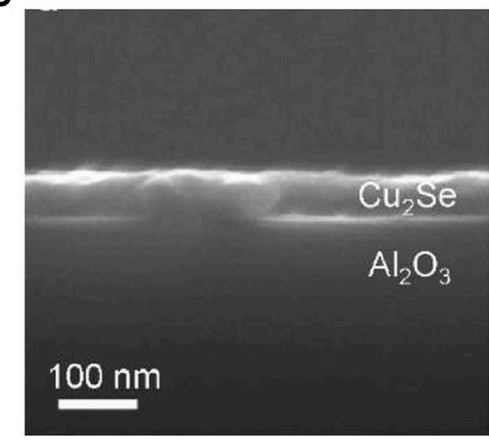

E

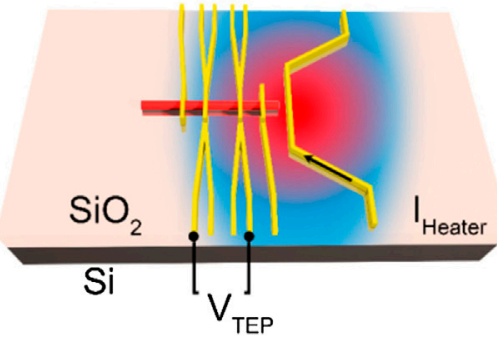

B

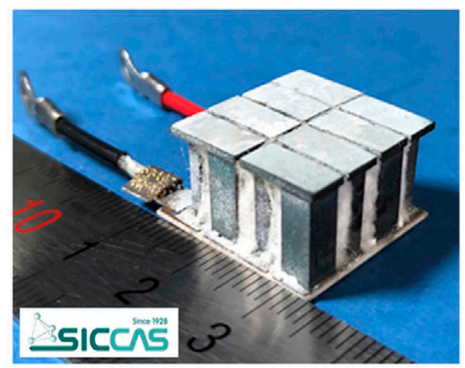

D

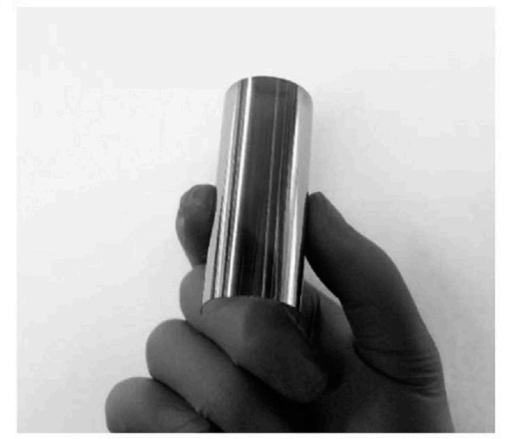

$\mathbf{F}$

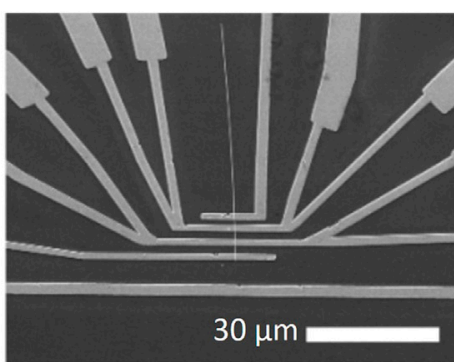

Figure 2 | (A) $\mathrm{Cu}_{2} \mathrm{~S}$ leg at a current density of $24 \mathrm{~A} \mathrm{~cm}^{-2}$ for $24 \mathrm{~h}$ and $\mathrm{Cu}_{1.8} \mathrm{~S}$ and CuS legs both after a stress of $48 \mathrm{~A} \mathrm{~cm}^{-2}$ for $72 \mathrm{~h}$ (from the left to right). Reproduced with permission (Dennler et al., 2014) Copyright 2014, Wiley-VCH. (B) Optical image of the eight-couple $\mathrm{Cu}_{2} \mathrm{Se}_{\mathrm{Nb}} \mathrm{Yb}_{0.3} \mathrm{CO}_{4} \mathrm{Sb}_{12} \mathrm{TE}_{\mathrm{modul}}$. Reproduced with permission (Qiu et al., 2019) Copyright 2019, Elsevier Inc. (C) Cross-sectional scanning electron microscope (SEM) image of the $\mathrm{Cu}_{2} \mathrm{Se}_{\text {film }}$ on $\mathrm{Al}_{2} \mathrm{O}_{3}$ substrate. (D) Photograph of the $\mathrm{Cu}_{2} \mathrm{Se}$ thin film on the polyimide substrate. Reproduced with permission (Lin et al., 2017). Copyright 2017, Wiley-VCH. (E) The illustration for the TE properties measurement for $\mathrm{CdS} / \mathrm{Cu}_{2} \mathrm{~S}$ superlattice nanowire. (F) SEM image of the fabricated device for thermopower measurement. Reproduced with permission (Xiong et al., 2017). Copyright 2017, American Chemical Society.

\section{CONCLUSIONS AND PERSPECTIVES}

Significant developments and progresses have been achieved in TE performance of binary copper chalcogenides. PLEC concept opens up a new situation to explain the low $\kappa$ in copper chalcogenide superlattice structure. Furthermore, tuning compositions optimizes $n$ leading to improved TE parameters. The main challenges for copper chalcogenide TE materials include the electrical and thermal stabilities during hightemperature measurement and the application limitations of the bulk modulus. It is hoped that binary copper chalcogenides will be widely applied in TE generators and device applications.

\section{AUTHOR CONTRIBUTIONS}

All authors listed have made a substantial, direct, and intellectual contribution to the work, and approved it for publication.

\section{ACKNOWLEDGMENTS}

The authors thank the National Natural Science Foundation of China (51702091, 51702046); the College Outstanding Young Scientific and Technological Innovation Team of Hubei province (T201922); and the Special Funding of Preventing the spread of COVID-19, Hubei University of Education (20XGZX20). 


\section{REFERENCES}

Chen, X., Yang, J., Wu, T., Li, L., Luo, W., Jiang, W., et al. (2018). Nanostructured binary copper chalcogenides: synthesis strategies and common applications. Nanoscale 10(32), 15130-15163. doi:10.1039/c8nr05558k

Chen, X., Zhang, H., Zhao, Y., Liu, W.-D., Dai, W., Wu, T., et al. (2019). Carbonencapsulated copper sulfide leading to enhanced thermoelectric properties. ACS Appl. Mater. Interfaces. 11, 22457-22463. doi:10.1021/acsami.9b06212

Dennler, G., Chmielowski, R., Jacob, S., Capet, F., Roussel, P., Zastrow, S., et al. (2014). Are binary copper sulfides/selenides really new and promising thermoelectric materials? Adv. Energy Mater. 4(9), 1301581. doi:10.1002/ aenm.201301581

Gahtori, B., Bathula, S., Tyagi, K., Jayasimhadri, M., Srivastava, A. K., Singh, S., et al. (2015). Giant enhancement in thermoelectric performance of copper selenide by incorporation of different nanoscale dimensional defect features. Nano Energy. 13, 36-46. doi:10.1016/j.nanoen.2015.02.008

Ge, Z.-H., Zhao, L.-D., Wu, D., Liu, X., Zhang, B.-P., Li, J.-F., et al. (2016). Low-cost, abundant binary sulfides as promising thermoelectric materials. Mater. Today. 19(4), 227-239. doi:10.1016/j.mattod.2015.10.004.10.1016/j.mattod.2015.10.004

He, Y., Day, T., Zhang, T., Liu, H., Shi, X., Chen, L., et al. (2014). High thermoelectric performance in non-toxic earth-abundant copper sulfide. Adv. Mater. 26(23), 3974-3978. doi:10.1002/adma.201400515

He, Y., Lu, P., Shi, X., Xu, F., Zhang, T., Snyder, G. J., et al. (2015a). Ultrahigh thermoelectric performance in mosaic crystals. Adv. Mater. 27(24), 3639-3644. doi:10.1002/adma.201501030

He, Y., Zhang, T., Shi, X., Wei, S.-H., and Chen, L. (2015b). High thermoelectric performance in copper telluride. NPG Asia Mater. 7(8), e210. doi:10.1038/am. 2015.91

Lin, Z., Hollar, C., Kang, J. S., Yin, A., Wang, Y., Shiu, H.-Y., et al. (2017). A solution processable high-performance thermoelectric copper selenide thin film. Adv. Mater. 29(21), 1606662. doi:10.1002/adma.201606662

Liu, H., Shi, X., Xu, F., Zhang, L., Zhang, W., Chen, L., et al. (2012). Copper ion liquid-like thermoelectrics. Nature Mater. 11(5), 422-425. doi:10.1038/ nmat3273

Liu, H., Yuan, X., Lu, P., Shi, X., Xu, F., He, Y., et al. (2013). Ultrahigh thermoelectric performance by electron and phonon critical scattering in Cu2Se1-xIx. Adv. Mater. 25(45), 6607-6612. doi:10.1002/adma.201302660

Mao, T., Qiu, P., Du, X., Hu, P., Zhao, K., Xiao, J., et al. (2019a). Enhanced thermoelectric performance and service stability of $\mathrm{Cu} 2 \mathrm{Se}$ via tailoring chemical compositions at multiple atomic positions. Adv. Funct. Mater. 30(6), 1908315. doi:10.1002/adfm.201908315

Mao, T., Qiu, P., Hu, P., Du, X., Zhao, K., Wei, T. R., et al. (2019b). Decoupling thermoelectric performance and stability in liquid-like thermoelectric materials. Adv. Sci. 7(1), 1901598. doi:10.1002/advs.201901598

Nunna, R., Qiu, P., Yin, M., Chen, H., Hanus, R., Song, Q., et al. (2017). Ultrahigh thermoelectric performance in Cu2Se-based hybrid materials with highly dispersed molecular CNTs. Energy Environ. Sci. 10, 1928-1935. doi:10.1039/ c7ee01737e
Qiu, P., Agne, M. T., Liu, Y., Zhu, Y., Chen, H., Mao, T., et al. (2018). Suppression of atom motion and metal deposition in mixed ionic electronic conductors. Nat. Commun. 9(1), 2910. doi:10.1038/s41467-018-05248-8

Qiu, P., Mao, T., Huang, Z., Xia, X., Liao, J., Agne, M. T., et al. (2019). Highefficiency and stable thermoelectric module based on liquid-like materials. Joule 3(6), 1538-1548. doi:10.1016/j.joule.2019.04.010

Qiu, P., Shi, X., and Chen, L. (2016). Cu-based thermoelectric materials. Energy Storage Mater. 3, 85-97. doi:10.1016/j.ensm.2016.01.009

Service, R. F. (2004). THERMOELECTRICS: temperature rises for devices that turn heat into electricity. Science 306, 806-807. doi:10.1126/science.306.5697.806

Shi, X., Chen, L., and Uher, C. (2016). Recent advances in high-performance bulk thermoelectric materials. Int. Mater. Rev. 61(6), 379-415. doi:10.1080/ 09506608.2016.1183075

Tan, G., Zhao, L.-D., and Kanatzidis, M. G. (2016). Rationally designing highperformance bulk thermoelectric materials. Chem. Rev. 116(19), 12123-12149. doi:10.1021/acs.chemrev.6b00255

Tang, H., Sun, F.-H., Dong, J.-F., AsfandiyarZhuang, H.-L., Pan, Y., et al. (2018) Graphene network in copper sulfide leading to enhanced thermoelectric properties and thermal stability. Nano Energy. 49, 267-273. doi:10.1016/j. nanoen.2018.04.058

Wei, T.-R., Qin, Y., Deng, T., Song, Q., Jiang, B., Liu, R., et al. (2018). Copper chalcogenide thermoelectric materials. Sci. China Mater. 62(1), 8-24. doi:10. 1007/s40843-018-9314-5

Xiong, Z., Cai, Y., Ren, X., Cao, B., Liu, J., Huo, Z., et al. (2017). Solution-processed $\mathrm{CdS} / \mathrm{Cu} 2 \mathrm{~S}$ superlattice nanowire with enhanced thermoelectric property. ACS Appl. Mater. Interfaces. 9(38), 32424-32429. doi:10.1021/acsami.7b09346

Zhao, K., Liu, K., Yue, Z., Wang, Y., Song, Q., Li, J., et al. (2019). Are Cu 2 Te-based compounds excellent thermoelectric materials? Adv. Mater. 31(49), 1903480. doi:10.1002/adma.201903480

Zhou, X., Yan, Y., Lu, X., Zhu, H., Han, X., Chen, G., et al. (2018). Routes for highperformance thermoelectric materials. Mater. Today. 21(9), 974-988. doi:10. 1016/j.mattod.2018.03.039

Zhu, G., Zhang, F., Li, X., Luo, W., Li, L., Zhang, H., et al. (2019). Engineering the distribution of carbon in silicon oxide nanospheres at the atomic level for highly stable anodes. Angew. Chem. Int. Ed. 58(20), 6669-6673. doi:10.1002/anie. 201902083

Conflict of Interest: The authors declare that the research was conducted in the absence of any commercial or financial relationships that could be construed as a potential conflict of interest.

Copyright (c) 2020 Chen, Yang, Katkus, Wu, Tao, Li, Wang, Wang and Dai. This is an open-access article distributed under the terms of the Creative Commons Attribution License (CC BY). The use, distribution or reproduction in other forums is permitted, provided the original author(s) and the copyright owner(s) are credited and that the original publication in this journal is cited, in accordance with accepted academic practice. No use, distribution or reproduction is permitted which does not comply with these terms. 